\title{
Dkk-3 as a potential biomarker for diagnosis and prognosis of colorectal cancer
}

\author{
Elahe Safari ${ }^{1,2}$, Ghasem Mosayebi ${ }^{3,4 *}$, Samaneh Khorrami ${ }^{1,2 *}$ \\ Received: 3 Dec 2017 \\ Published: 14 Sep 2018
}

\begin{abstract}
Background: The Dickkopf 3 (Dkk-3) protein is a member of the Dkk family known as Wnt signaling inhibitor. The level of DKk-3 changes in a wide range of cancers, such as colorectal cancer, lung cancer, prostate cancer, and bladder cancer, is proposed as a biomarker for diagnosis and prognosis of many cancers. The present study was conducted to evaluate the serum level of Dkk-3 as a cancer biomarker and to determine their prognostic value in colorectal cancer (CRC) patients and healthy matched controls.

Methods: A total of 30 colorectal cancer patients at different stages of the disease and healthy matched controls with no history of inflammatory and autoimmune disease or cancer were enrolled in the study. The level of Dkk-3 was assessed serologically using enzymelinked immunosorbent assay (ELISA) method, moreover, relevance of these markers with patients' clinicopathological features was subsequently assessed. Means comparison and ROC curves analysis were done using SPSS software. P-value $<0.05$ was considered significant in all the tests.

Results: In this study, it was revealed that serum level of Dkk-3 was significantly $(\mathrm{p}<0.001)$ lower in patients compared to the healthy controls. Statistical analysis showed that serum level of Dkk-3 has $78 \%$ specificity and $77 \%$ sensitivity (AUC $=0.782,95 \% \mathrm{CI}$ ) for diagnosis of colorectal cancer.

Conclusion: Dkk-3 protein can be considered as a potential biomarker for diagnosis and possibly the prognosis of colorectal cancer.
\end{abstract}

Keywords: Dkk-3, Colorectal cancer, Biomarker

Copyright $\odot$ Iran University of Medical Sciences

Cite this article as: Safari E, Mosayebi Gh, Khorrami S. Dkk-3 as a potential biomarker for diagnosis and prognosis of colorectal cancer. Med J Islam Repub Iran. 2018 (14 Sep);32:86. https://doi.org/10.14196/mjiri.32.86

\section{Introduction}

Colorectal cancer (CRC) is the third most common cause of death worldwide (1-5). CRC incidence rate has increased in developing countries, including Iran and other Asian countries, due to changes related to culture and eating habits (3, 6-9). In most patients with colorectal cancer, the disease is asymptomatic at early stages; thus, many screening tools and molecular biomarkers are developed to detect colorectal cancer in early stages.

Dickkopf (Dkk) encodes 4 major secreted glycoproteins (Dkk1,2,3,4) as negative regulators of Wnt signaling, which involve 255 to 350 amino acids with a molecular

Corresponding author: Dr. Samaneh Khorrami, khorami.s@iums.ac.ir Dr. Ghasem Mosayebi, ghasemmosayebi@arakmu.ac.ir

1. Department of Immunology, School of Medicine, Iran University of Medical Sciences, Tehran, Iran.

2. Immunology Research Center, Institute of Immunology and Infectious Disease, Iran University of Medical Sciences, Tehran, Iran.

3. Molecular and Medicine Research Center, Arak University of Medical Sciences, Arak Iran.

4. Department of Immunology, School of Medicine, Arak University of Medical Sciences, Arak, Iran weight of 24 and $29 \mathrm{kDa}$ (Dkk1, 2 and -4$)$ and $38 \mathrm{kDa}$ (Dkk3) $(10,11)$.

Dkk-3 or REIC (reduced expression in immortalized cells) functions as a growth suppressor during human embryonic development $(10,12,13)$. This factor can decrease the cytoplasmic accumulation of $\beta$-catenin and can also impede the activity of T-cell factor 4 , showing its anticancer property $(13,14)$. According to previous studies, some cancers, such as lung cancer, gastric cancer, ovarian cancer, acute lymphoblastic leukemia, prostate cancer and bladder

$\uparrow$ What is "already known" in this topic:

Some cancers, such as lung cancer, gastric cancer, ovarian cancer and etc. have lower levels of DKK-3, as Wnt signaling inhibitor. However, tumor endothelium of colorectal cancer has shown higher levels of DKK-3. Researchers pay more attention to the potential use of Dkk-3 as a therapeutic target that can inhibit cancer cell growth to suppress tumors.

\section{$\rightarrow$ What this article adds:}

DKK-3 in the serum of colorectal cancer patients, was lower in patients compared to healthy controls. DKK-3 have higher sensitivity and specificity with higher ROC-AUC values for DKK3. Our results showed a lower level of circulating Dkk-3 in the serum level of patients with lymph and vascular invasion compared to the group with no lymph and vascular invasion. 
cancer, have lower levels of DKK-3 (14-18). However, tumor endothelium of colorectal cancer has shown higher levels of DKK-3 (19). Regarding carcinogenesis of colorectal cancer, further researches are needed to determine the serum levels of Dkk-3, whose role is probably through Wnt/beta-catenin pathway. Nowadays, researchers pay more attention to the potential use of Dkk-3 as a therapeutic target that can inhibit cancer cell growth to suppress tumors.

Based on investigations in assessing DKK3 expression, this protein may have a cancer-specific expression pattern and a potential alternative role in cancer invasion. In the present study, we aimed at investigating the serum level of Dkk-3 in patients with different stages of colon cancer compared to the healthy group. We also evaluated the serum levels of Dkk-3 separation based on clinicopathological features of patients.

\section{Methods \\ Study population}

In the present study, serum samples were obtained from 30 patients with colorectal cancer and 28 healthy individuals without any family history of cancer, inflammatory diseases, or autoimmune diseases who referred to Iran $\mathrm{Na}$ tional Tumor Bank, Imam Khomeini Hospital, Tehran, Iran. All patients provided written consent. The study was approved by the institutional ethics committee of the Cancer Institute of Tehran and in accordance with the declaration of Helsinki.

Samples were selected considering the following criteria according to a previous study: (i) patients not receiving any anticancer treatment, and (ii) those patients whose tumors were pathologically diagnosed as colorectal cancer (stage I-IV). An expert oncologist determined the stage and grade tissue samples of patients according to guidelines and classifications. All data related to these patients were obtained from Iran National Tumor Bank, Imam Khomeini Hospital, Tehran, Iran, and were confirmed by immunohistochemistry (IHC).

\section{Blood sample collection and serum separation}

To collect serum samples, whole blood from 30 newly diagnosed patients and 28 healthy individuals was collected in untreated test tubes. After incubation at room temperature for 20 minutes, centrifugation was done at $1200 \mathrm{~g}$ for 10 minutes at $4^{\circ} \mathrm{C}$; then, supernatant (serum) was aliquoted and stored at $-80^{\circ} \mathrm{C}$.

\section{Serum DKK-3 measurement}

Levels of Dkk-3 in serum samples were evaluated by enzyme-linked immunosorbent assay (ELISA) methods. ELISA protocol was done based on the manufacturer's instructions (Raybiotech, Atlanta, USA). All samples were tested by duplication. Detection limits of Dkk-3 were 50 $\mathrm{pg} / \mathrm{mL}$. The threshold for Dkk-3 was determined based on ROC-AUC plot. Following the analysis, $81.78 \mathrm{pg} / \mathrm{mL}$ was selected as the cut-off point for Dkk-3.

\section{Statistical analysis}

The mean \pm standard error of the mean (SEM), median, and other statistical values of Dkk-3 were measured. Independent samples $t$ test and ANOVA with Tukey's post hoc tests were used to measure differences between levels of Dkk-3 in the sera of patients and controls, along with between-groups comparison. Sensitivity, specificity, positive predictive values (PPV), and negative predictive values (NPV) were computed to evaluate the diagnostic performance of Dkk-3. ROC (Receiver Operator Characteristic) curves and AUC (areas under the curve) with 95\% CI (confidence interval) were analyzed by SPSS software. P-value $<0.05$ was considered significant for all the tests.

\section{Results}

\section{Characteristic of included samples}

We included 30 patients with colon cancer who referred to Iran Tumor Bank, Imam Khomeini hospital in Tehran. Also, 28 healthy individuals were tested whose clinicopathological features are summarized in Table 1 . The mean age of colon cancer patients was 57 years, which was matched with that of the controls (mean age: 55). Overall, 6 cases $(21.43 \%)$ had stage I, $9(30 \%)$ stage II, $10(33 / 33 \%)$ stage III, and $5(16.66 \%)$ stage IV colon cancer.

Also, the number of lymphatic invasion in positive and/or negative cases was $17(56.6 \%)$ and $13(43.3 \%)$, and the number of vascular invasions in positive and/or negative cases was $16(53.3 \%)$ and $14(46.6 \%)$, respectively.

\begin{tabular}{lll}
\multicolumn{3}{l}{ Table 1. The clinicopathological features of patients } \\
\hline Descriptive data & Patient & Control \\
\hline $\begin{array}{l}\text { Number of cases } \\
\text { Gender, N }\end{array}$ & 30 & 28 \\
Female & 17 & 18 \\
Male & 13 & 10 \\
Median age, yrs & $57 \pm 11.9$ & $55 \pm 8.2$ \\
(range) & & \\
Vascular invasion, N & 16 & - \\
Lymphatic invasion, & 17 & - \\
N & & \\
Dkk3 (me- & $54.27 \pm 7$ & $101.96 \pm 9$ \\
dian+SEM) & & \\
\hline
\end{tabular}

\begin{tabular}{ccc}
\multicolumn{3}{c}{ Table 2. Serum levels of markers based on FIGO stage } \\
\hline Stages & Number of cases & Dkk-3 (Mean \pm SEM) \\
\hline Grade 1 & 6 & $49.48 \pm 16$ \\
Grade 2 & 9 & $51.15 \pm 13$ \\
Grade 3 & 10 & $49.53 \pm 13$ \\
Grade 4 & 5 & $74.69 \pm 9$ \\
control & 28 & $101.96 \pm 9$ \\
\hline
\end{tabular}

Table 3. Serum levels of markers based on vascular and lymphatic invasion.

\begin{tabular}{lcc}
\hline & $\begin{array}{c}\text { Number of } \\
\text { cases }\end{array}$ & $\begin{array}{c}\text { Dkk-3 } \\
\text { (Mean } \pm \text { SEM) }\end{array}$ \\
\hline Vascular invasion + & 17 & $46.20 \pm 8.35$ \\
Vascular invasion - & 11 & $68.74 \pm 13.27$ \\
Lymphatic invasion & 16 & $35.19 \pm 8.7$ \\
+ & & \\
Lymphatic invasion & 13 & $65.06 \pm 11.65$ \\
- & & \\
\hline
\end{tabular}



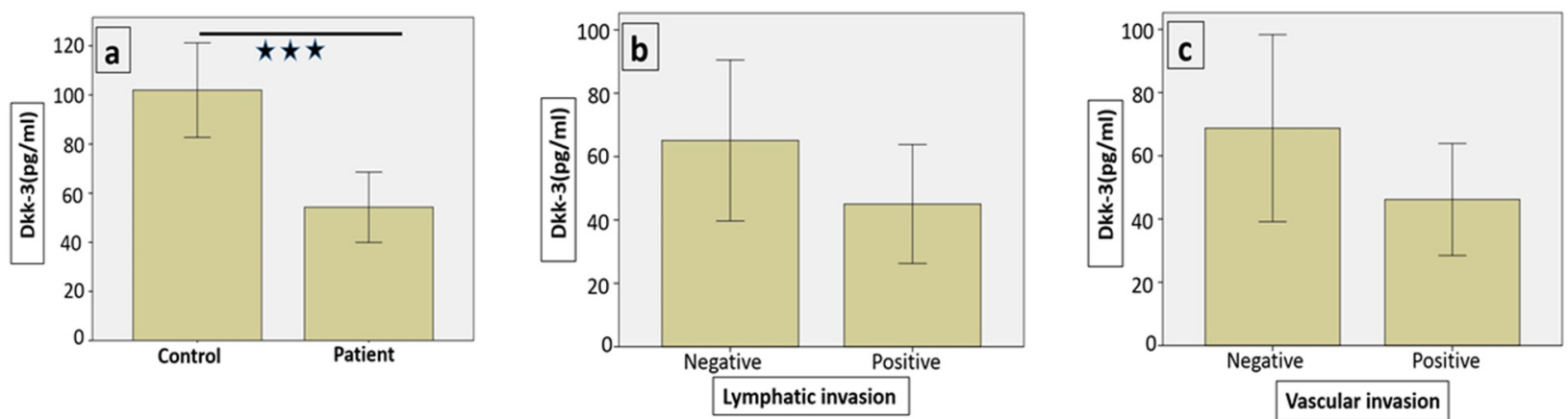

Fig. 1. The serum level of Dkk-3 in patients and controls

The serum level of Dkk-3 was lower in patients comparing to controls $(\mathrm{P}<0.001)$. Serum levels of Dkk-3 patients separated based on lymphatic invasion (b) Serum levels of Dkk-3 patients separated based on vascular invasion (c). $(*=\mathrm{P}<0.05, * *=\mathrm{P}<0.01, * * *=\mathrm{P}<0.001)$
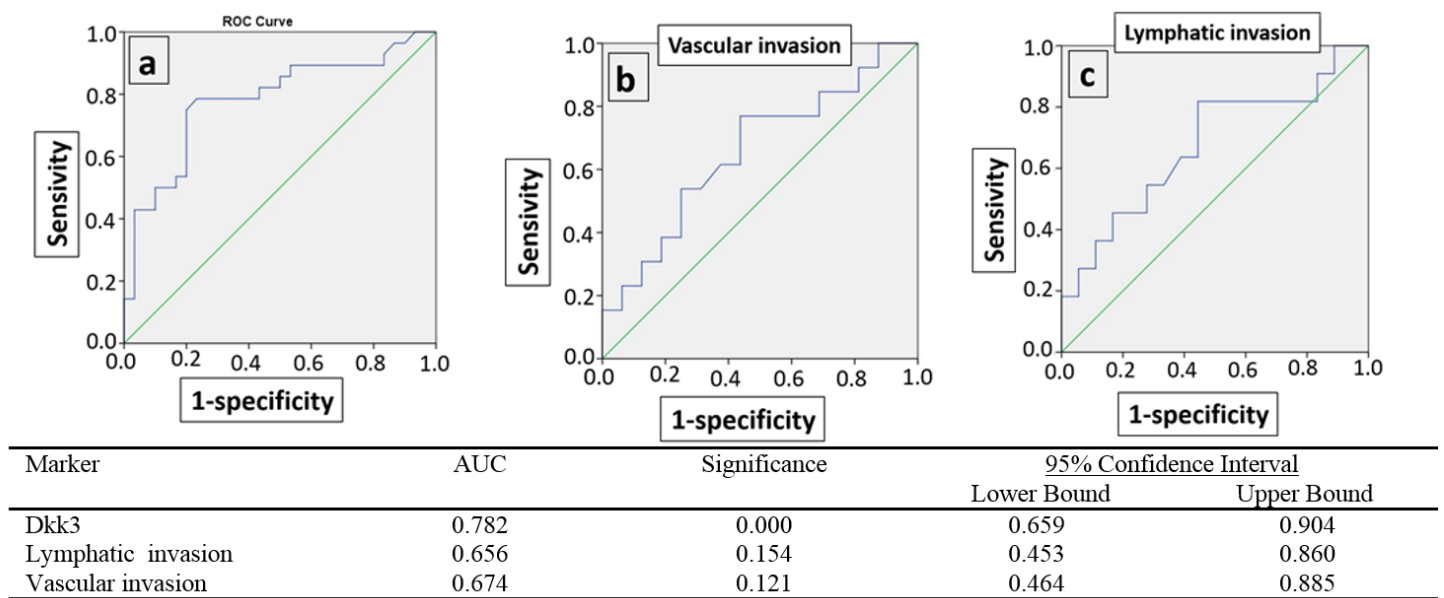

\begin{tabular}{lcccc}
\hline Marker & AUC & Significance & \multicolumn{3}{c}{ 95\% Confidence Interval } \\
\cline { 3 - 5 } & & & Lower Bound & Upper Bound \\
\hline Dkk3 & 0.782 & 0.000 & 0.659 & 0.904 \\
Lymphatic invasion & 0.656 & 0.154 & 0.453 & 0.860 \\
Vascular invasion & 0.674 & 0.121 & 0.464 & 0.885 \\
\hline
\end{tabular}

Fig. 2. ROC plot and AUC for Dkk-3. ROC plot and AUC for Dkk-3 for patients and controls(a), for lymphatic invasion (b) and lymphatic inva$\operatorname{sion}(\mathrm{c})$.

\section{Serum levels of DKK-3}

The serum level of DKK-3 heterodimer was assessed using ELISA method in patients and controls according to the manufacturer's instructions. The serum levels of DKK-3 in colon cancer patients and healthy controls were $54.27 \pm 7.00$ and $101.96 \pm 9.37 \mathrm{pg} / \mathrm{mL}$, respectively $(\mathrm{p}<0.001)$. Serum levels of DKK-3 in patients, with regards to the TNM staging of cancer, are summarized in Table 2. Our data revealed that the serum level of DKK-3 is significantly lower in patients compared to the healthy controls $(\mathrm{p}<0.001)$ (Fig. 1a).

However, the level of circulating DKK-3 showed no significant differences in patients at different stages. Moreover, despite the lower level of DKK-3 observed in those patients who were positive for lymph and vascular invasion, this difference was not statistically significant compared to the negative group ( $\mathrm{p}>0.05$ ) (Table 3 ) (Fig. 1 b,c).

\section{Predictive performance of DKK-3}

Sensitivity, specificity, PPV, and NPV values for DKK-
3 are presented in Table 4 . The receiver operating characteristic (ROC) plot and AUC for DKK-3 were performed (Fig. 2 a). The AUC (the area under the ROC curve) for DKK-3 was significantly high (0.78), and it could distinguish between patients and healthy individuals $(\mathrm{p}<0.001)$. In addition, ROC-AUC plot for DKK-3 for the lymph and vascular invasion indicated that the predictive performance of DKK-3 for prognostic value was not promising (Fig. 2 b, c).

\section{Discussion}

Over the last decade, there has been an outstanding improvement in our understanding of the characteristics of colon cancer; yet, in nearly $50 \%$ of the cases, they are identified in asymptomatic persons through colonoscopy or CT colonography performed for reasons other than colon cancer (20). There are several screening tests available to detect colon cancer, but none is completely reliable although a good serum marker can strongly improve the speed and authenticity of the screening process. In this case-control

Table 4. Sensitivity, specificity, PPV, and NPV of markers in patients and controls

\begin{tabular}{lcccc}
\hline Marker & Sensitivity $\%$ & Specificity $\%$ & PPV \% & NPV \% \\
\hline Dkk-3 & 77 & 78 & 78.6 \\
Cut-off points Dkk-3 $=81.7897 \mathrm{pg} / \mathrm{mL}$ & & & \\
\hline
\end{tabular}


study, we investigated the capability of Dkk-3 as a new biomarker to predict colon cancer development. Dkk-3, a protein biomarker involved in activating genes whose expression elevates angiogenesis, tumor migration, and premetastatic niche formation (21), may be a useful biomarker.

Our data showed that DKK-3 values have substantial differences between patients and controls. According to our data, sensitivity and specificity of DKK-3 are strongly high. Fung et al. reported ROC-AUC values for DKK-3, which was 0.69 (22). Our data for the Dkk-3 protein showed higher AUC (0.78) compared to these results.

Yang et al. have shown that downregulation in mRNA and protein level can be seen in the expression level of DKK3 in colorectal adenocarcinoma cell lines. G0/G1 cell cycle arrest, induced apoptosis, lower cell proliferation, and high cytoplasmic beta-catenin are some of the consequences of forced overexpression of DKK3 mRNA (23). Also, it has been demonstrated that the expression level of DKK3 protein has been reduced in colorectal tumor tissue compared to the normal tissues, which is associated with invasion depth, TNM stage, and dedifferentiation of colorectal cancer $(21,24)$.

On the other hand, we investigated the possibility that these biomarkers may distinguish between stages of cancer, assuming that a screening biomarker can diagnose tumor development in its early stages. According to our results, there were no considerable differences between the stages of cancer in both investigated biomarkers, the ROC-AUC was low for all stages, and the p-value was not significant. However, this could have been due to a small number of patients with a different stage of colon cancer.

Previous studies demonstrated the correlation of Dkk3 expression and prevention of tumor invasion.

Maehata et al. found that the siRNA-mediated Dkk-3 silencing caused an elevation in the invasion capacity in colon cancer SW480 cells (25). Also, Saeb-Party et al. reported the reduced invasion of Matrigel $\AA$ based assays and observed delayed wound healing in scratch assays in urothelial cell carcinoma EJ28 cells treated with Dkk-3 conditioned medium (26). Also, similar results were shown in a study by Edamura et al. that was performed in orthotopic mouse models of prostate cancer, malignant mesothelioma, and scirrhous gastric carcinoma. On the other hand, it was shown that the Dkk-3 gene vector injection into the tumor in the first model can prevent the prostate tumor growth and lymph node metastasis. The tumor tissues had high amounts of apoptosis and reduction in Dkk-3-mediated invasion capacity, and in vitro assays demonstrated the inhibitory effect on metastasis. Furthermore, a high survival rate was found among the mice administered by Dkk3 gene as a result of anti-tumor effects (27).

However, investigations on the role of DKK3 in angiogenesis researchers have shown that in tumor endothelium of colorectal cancer (rank \#4), DKK3 gene was strongly upregulated in accordance with RNA expression analyses on purified endothelial cells, which is in line with the protein level assay $(28,29)$. Also, the vessels adjacent to the cancer tissue have shown DKK3 protein expression in immunohistochemical analysis, indicating the proangiogenic function of the DKK3 protein $(28,29)$.

Thus, despite growth inhibitor on epithelium originated tumor cells, Dkk-3 can attribute to differentiation of endothelial cells in the tumor, representing the proangiogenic effect of Dkk-3 in vascularization and possibility of being a marker for neo-angiogenesis (29). Glioma, non-Hodgkin's lymphomas with high grade, melanoma (28), gastric cancer (30), prostate cancer (31), and pancreas cancer (32) have shown an elevated number of blood vessels expressing Dkk-3 than normal tissues. According to a study reporting higher expression of Dkk-3 in blood vessels of low-grade adenocarcinomas in pancreatic tissue, Dkk-3 protein might be a differentiation factor for remodeling the tumor vasculature (32). However, this is inconsistent with observations in gastric cancer (30). The expression of Dkk-3 protein had no influence on primary ECFC (endothelial colony-forming cells) during in vitro study on cell proliferation and migration assays. The Dkk-3 downregulation caused a reduction in tube formation in Matrigel ${ }^{\circledR}$, which was elevated after enforced overexpression (28). Moreover, microvessel density was significantly enhanced in the C57/BL6 melanoma mouse model after high expression of Dkk-3 in B16F10 melanoma cells (28). Thus, one of the most important pro-angiogenic proteins in neovascularization and markers for neo-angiogenesis in CRC has been reported to be Dkk-3 (29).

However, our results have shown a lower level of circulating Dkk-3 in the serum level of patients with lymph and vascular invasion compared to the negative group without lymph and vascular invasion, but this difference was not statistically significant. This might be because of a low number of samples in this study. Thus, further studies should be conducted on a larger number of CRC patients with different clinicopathological features.

\section{Conclusion}

In conclusion, the lower level of circulating of Dkk3 in the serum of colon cancer patients might provide evidence for supporting the potential role of Dkk3 as a tumor marker for diagnosis, prognosis, and monitoring of CRC, as statistical analysis revealed that serum level of Dkk-3 has 78\% specificity and $77 \%$ sensitivity for diagnosis of colorectal cancer. Little research has been done on the prognostic value of tumor markers, and thus the aim of our study was to achieve such valuable data. However, to evaluate colon cancer biomarkers, more comprehensive studies are required.

We suggest that DKK-3 protein be used as a biomarker in colon cancer, as it can perfectly identify CRC patients with high specificity and sensitivity.

\section{Acknowledgment}

This study was supported by School of Medicine, Arak University of Medical Sciences, Arak, Iran. All samples were documented by Iran National Tumor Bank, Imam Khomeini hospital.

\section{Conflict of Interests}

The authors declare that they have no competing interests. 


\section{References}

1. Becerra AZ, Probst CP, Tejani MA, Aquina CT, Gonzalez MG, Hensley BJ, et al. Evaluating the Prognostic Role of Elevated Preoperative Carcinoembryonic Antigen Levels in Colon Cancer Patients: Results from the National Cancer Database. Ann Surg Oncol. 2016;23(5):155461.

2. Siegel R, Desantis C, Jemal A. Colorectal cancer statistics, 2014. CA Cancer J Clin. 2014;64(2):104-17.

3. Dolatkhah R, Some MH, Kermani IA, Ghojazadeh M, Jafarabadi MA, Farassati $\mathrm{F}$, et al. Increased colorectal cancer incidence in Iran: a systematic review and meta-analysis. BMC Pub Health. 2015;15:997.

4. Mousavi SM, Gouya MM, Ramazani R, Davanlou M, Hajsadeghi N, Seddighi Z. Cancer incidence and mortality in Iran. Ann Oncol. 2009;20(3):556-63.

5. Ansari R, Mahdavinia M, Sadjadi A, Nouraie M, Kamangar F, Bishehsari F, et al. Incidence and age distribution of colorectal cancer in Iran: results of a population-based cancer registry. Cancer Lett.2006;240(1):143-7.

6. Nataraj SM, Prema CL, Vimalambike MG, Shivalingaiah SC, Sundaram S, Kumar AP, et al. Major Protein of Carcinoembryonic Antigen Gene Family - CD66c, A Novel Marker in Colon Carcinoma. J Clin Diagn Res. 2016;10(2):XC01-XC4.

7. Dong Y, Yu J, Ng SS. MicroRNA dysregulation as a prognostic biomarker in colorectal cancer. Cancer Manag Res. 2014;6:405-22.

8. Zhou F, Mu YD, Liang J, Liu ZX, Chen HS, Zhang JF. Expression and prognostic value of tumor stem cell markers ALDH1 and CD133 in colorectal carcinoma. Oncol Lett. 2014;7(2):507-12.

9. Thenappan A, Li Y, Shetty K, Johnson L, Reddy EP, Mishra L. New Therapeutics Targeting Colon Cancer Stem Cells. Current Colorect Cancer Rep. 2009;5(4):209.

10.Krupnik VE, Sharp JD, Jiang C, Robison K, Chickering TW, Amaravadi $\mathrm{L}$, et al. Functional and structural diversity of the human Dickkopf gene family. Gene. 1999;238(2):301-13.

11.Niehrs C. Function and biological roles of the Dickkopf family of Wnt modulators. Oncogene. 2006;25(57):7469-81.

12.Glinka A, Wu W, Delius H, Monaghan AP, Blumenstock C, Niehrs C. Dickkopf-1 is a member of a new family of secreted proteins and functions in head induction. Nature. 1998;391(6665):357-62.

13. Hoang BH, Kubo T, Healey JH, Yang R, Nathan SS, Kolb EA, et al. Dickkopf 3 inhibits invasion and motility of Saos-2 osteosarcoma cells by modulating the Wnt-beta-catenin pathway. Cancer Res. 2004;64(8):2734-9.

14.Yue W, Sun Q, Dacic S, Landreneau RJ, Siegfried JM, Yu J, et al. Downregulation of Dkk3 activates beta-catenin/TCF-4 signaling in lung cancer. Carcinogenesis. 2008;29(1):84-92.

15.Chim CS, Pang R, Liang R. Epigenetic dysregulation of the Wnt signalling pathway in chronic lymphocytic leukaemia. J Clin Pathol. 2008;61(11):1214-9.

16.Kurose K, Sakaguchi M, Nasu Y, Ebara S, Kaku H, Kuriyama R, et al. a Decreased expression of REIC/Dkk-3 in human renal clear cell carcinoma. J Urol. 2004;171(3):1314-8.

17.Ueno K, Hirata H, Majid S, Chen Y, Zaman MS, Tabatabai ZL, et al. Wnt antagonist DICKKOPF-3 (Dkk-3) induces apoptosis in human renal cell carcinoma. Mol Carcinog. 2011;50(6):449-57.

18. Veeck J, Bektas N, Hartmann A, Kristiansen G, Heindrichs U, Knuchel R, et al. Wnt signaling in human breast cancer: expression of the putative Wnt inhibitor Dickkopf-3 (DKK3) is frequently suppressed by promoter hypermethylation in mammary tumors. Breast Cancer Res. 2008;10(5): R82.

19.St Croix B, Rago C, Velculescu V, Traverso G, Romans KE, Montgomery $\mathrm{E}$, et al. Genes expressed in human tumor endothelium. Science. 2000;289(5482):1197-202.

20.Strum WB. Colorectal Adenomas. N Engl J Med. 2016;374(11):106575.

21.Veeck J, Dahl E. Targeting the Wnt pathway in cancer: the emerging role of Dickkopf-3. Biochim. Biophys. Acta. 2012;1825(1):18-28.

22.Fung KY, Tabor B, Buckley MJ, Priebe IK, Purins L, Pompeia C, et al. Blood-based protein biomarker panel for the detection of colorectal cancer. PLoS ONE. 2015;10(3):e0120425.

23. Yang ZR, Dong WG, Lei XF, Liu M, Liu QS. Overexpression of Dickkopf-3 induces apoptosis through mitochondrial pathway in human colon cancer. World J Gastroenterol. 2012;18(14):1590-601.

24.Wang W, Zhu W, Xu XY, Nie XC, Yang X, Xing YN, et al. The clini- copathological significance of REIC expression in colorectal carcinomas. Histol Histopathol. 2012;27(6):735-43.

25.Maehata T, Taniguchi H, Yamamoto H, Nosho K, Adachi Y, Miyamoto N, et al. Transcriptional silencing of Dickkopf gene family by $\mathrm{CpG}$ island hypermethylation in human gastrointestinal cancer. World J Gastroenterol. 2008;14(17):2702-14.

26.Saeb-Parsy K, Veerakumarasivam A, Wallard MJ, Thorne N, Kawano Y, Murphy G, et al. MT1-MMP regulates urothelial cell invasion via transcriptional regulation of Dickkopf-3. Br J Cancer. 2008;99(4):6639 .

27.Edamura K, Nasu Y, Takaishi M, Kobayashi T, Abarzua F, Sakaguchi $\mathrm{M}$, et al. Adenovirus-mediated REIC/Dkk-3 gene transfer inhibits tumor growth and metastasis in an orthotopic prostate cancer model. Cancer Gene Ther. 2007;14(9):765-72.

28.Untergasser G, Steurer M, Zimmermann M, Hermann M, Kern J, Amberger A, et al. The Dickkopf-homolog 3 is expressed in tumor endothelial cells and supports capillary formation. Int $J$ Cancer. 2008;122(7):1539-47.

29.Zitt M, Untergasser G, Amberger A, Moser P, Stadlmann S, Zitt M, et al. Dickkopf-3 as a new potential marker for neoangiogenesis in colorectal cancer: expression in cancer tissue and adjacent non-cancerous tissue. Dis Markers. 2008;24(2):101-9.

30.Muhlmann G, Untergasser G, Zitt M, Zitt M, Maier H, Mikuz G, et al. Immunohistochemically detectable dickkopf-3 expression in tumor vessels predicts survival in gastric cancer. Virchows Arch. 2010;456(6):635-46.

31.Zenzmaier C, Untergasser G, Hermann M, Dirnhofer S, Sampson N, Berger P. Dysregulation of Dkk-3 expression in benign and malignant prostatic tissue. Prostate. 2008;68(5):540-7.

32.Fong D, Hermann M, Untergasser G, Pirkebner D, Draxl A, Heitz M, et al. Dkk-3 expression in the tumor endothelium: a novel prognostic marker of pancreatic adenocarcinomas. Cancer Sci. 2009;100(8):141420 . 